\title{
Analytical description of photon beam phase spaces in inverse Compton scattering sources
}

\author{
C. Curatolo, ${ }^{1, *}$ I. Drebot, ${ }^{1}$ V. Petrillo, ${ }^{1,2}$ and L. Serafini ${ }^{1}$ \\ ${ }^{1}$ INFN-Milan, via Celoria 16, 20133 Milano, Italy \\ ${ }^{2}$ Università degli Studi di Milano, via Celoria 16, 20133 Milano, Italy
}

(Received 9 March 2017; published 3 August 2017; publisher error corrected 14 September 2017)

\begin{abstract}
We revisit the description of inverse Compton scattering sources and the photon beams generated therein, emphasizing the behavior of their phase space density distributions and how they depend upon those of the two colliding beams of electrons and photons. The main objective is to provide practical formulas for bandwidth, spectral density, brilliance, which are valid in general for any value of the recoil factor, i.e. both in the Thomson regime of negligible electron recoil, and in the deep Compton recoil dominated region, which is of interest for gamma-gamma colliders and Compton sources for the production of multi-GeV photon beams. We adopt a description based on the center of mass reference system of the electron-photon collision, in order to underline the role of the electron recoil and how it controls the relativistic Doppler/boost effect in various regimes. Using the center of mass reference frame greatly simplifies the treatment, allowing us to derive simple formulas expressed in terms of rms momenta of the two colliding beams (emittance, energy spread, etc.) and the collimation angle in the laboratory system. Comparisons with Monte Carlo simulations of inverse Compton scattering in various scenarios are presented, showing very good agreement with the analytical formulas: in particular we find that the bandwidth dependence on the electron beam emittance, of paramount importance in Thomson regime, as it limits the amount of focusing imparted to the electron beam, becomes much less sensitive in deep Compton regime, allowing a stronger focusing of the electron beam to enhance luminosity without loss of mono-chromaticity. A similar effect occurs concerning the bandwidth dependence on the frequency spread of the incident photons: in deep recoil regime the bandwidth comes out to be much less dependent on the frequency spread. The set of formulas here derived are very helpful in designing inverse Compton sources in diverse regimes, giving a quite accurate first estimate in typical operational conditions for number of photons, bandwidth, spectral density and brilliance values - the typical figures of merit of such radiation sources.
\end{abstract}

DOI: 10.1103/PhysRevAccelBeams.20.080701

\section{INTRODUCTION}

Inverse Compton scattering sources (ICSs) are becoming increasingly attractive as radiation sources in photon energy regions either not covered by other high brilliance sources (FEL's, synchrotron light sources) or where compactness becomes an important figure of merit, like for advanced x-ray imaging applications to be implemented in university campus, hospitals, museums, etc., i.e. outside of research centers or large scale laboratories [1]. ICSs are becoming the $\gamma$-ray sources of reference in nuclear photonics, photo-nuclear [2,3] and fundamental physics [4], thanks to superior performances in spectral densities achievable. Eventually they will be considered for very high energy photon generation (in the $\mathrm{GeV}$ to $\mathrm{TeV}$ range) since there are no other competing techniques at present, neither on the horizon, based on

\footnotetext{
*orresponding author. camilla.curatolo@mi.infn.it

Published by the American Physical Society under the terms of the Creative Commons Attribution 4.0 International license. Further distribution of this work must maintain attribution to the author(s) and the published article's title, journal citation, and DOI.
}

artificial tools at this high photon energy [5]. As a consequence, a flourishing of design and commissioning activities is presently occurring in several laboratories [6-18] and companies [19-22], where ICSs are being conceived, designed and built to enable several domains of applications, and ranging from a few $\mathrm{keV}$ photon energy up to GeV's and beyond. Designs of ICSs are carried out considering several diverse schemes, ranging from high gradient room temperature pulsed rf linacs $[3,23,24]$ to super-conducting CW energy recovery linacs (ERL) $[25,26]$ or storage rings [2,27-30], as far as the electron beam generation is concerned, and from single pulse J-class amplified laser systems running at $100 \mathrm{~Hz}$ to optical cavities (e.g. Fabry-Perot) running at $100 \mathrm{MHz}$ acting as photon storage rings for the optical photon beams, not to mention schemes based on FEL's to provide the colliding photon beam [25,31,32].

In order to assess the performances of a specific ICSs under design, detailed simulations of the electron-photon beam collision are typically carried out using Monte Carlo codes [33-35] able to model the linear and nonlinear electronphoton quantum interaction leading to Compton back-scattering events, taking into account in a complete fashion the space-time propagation of the two colliding beams through the interaction point region, including possible multiple 
scattering events occurring during the overlap of the two pulses. Only in case of negligible electron recoil, i.e. in the so called Thomson regime typical of low energy X-ray ICSs, classical electromagnetic numerical codes (e.g. TSST [36]), modelling the equivalent undulator radiation emitted by electrons wiggling in the electromagnetic field of the incoming laser pulse, allow to analyze particular situations such as the use of chirped [37], tilted [38], and twisted [39] lasers.

In the recent past some efforts have been developed to carry out analytical treatments of the beam-beam collision physics, embedding the single electron-photon collision from a quantum point of view within a rms distribution of the scattered photon beam [30,40-46], or, within a classical framework, integrating the radiated power in the far field on the distributions of the colliding beams $[1,36,47]$. This latter approach suffers from a nonconservation of energy and momentum, due to its lack of describing correctly the electron recoil in the scattering process. We generalized the former approach to take into account in a complete fashion the recoil effect when averaging over the rms momenta of the two colliding beams, leading in this way to expressions for the bandwidth and spectral densities of the emitted photon beam which are valid for any scattering configuration, with the only restriction of considering relativistic electrons colliding head-on with photons of much smaller energy than electrons (typical of ICSs' operational conditions).

As extensively reported in the literature, the emitted photon beam formation is always accomplished by forward collimating the high energy back-scattered photons $[3,48]$, which are emitted all over the solid angle, though with a specific energy-angle correlation such that the most energetic photons propagate around the direction of motion of the electron beam within a small angle $\mathrm{O}(1 / \gamma)$, where $\gamma$ is the relativistic factor of the electron. As illustrated in Sec. II, carrying out the kinematics of the electron-photon collision in the center of mass reference system allows us to underline that the effective angle of collimation of high energy back-scattered photons is actually $1 / \gamma_{\mathrm{CM}}$, where $\gamma_{\mathrm{CM}}$ is the center of mass relativistic factor $\left(\gamma_{\mathrm{CM}}\right.$ is always smaller than $\gamma$, approaching $\gamma$ when the electron recoil tends to zero, i.e., in the Thomson regime). Using the center of mass reference frame the Doppler frequency enhancement of the scattered radiation can be recovered in the quantum treatment showing that the maximum energy of the scattered photons (the so-called Compton edge) can be expressed as $4 \gamma_{\mathrm{CM}}^{2}$ times the energy of the incident (optical) photon, therefore generalizing to deep Compton recoil regime the concept of Doppler/ boosted frequency enhancement due to the back-scattering process, which is a popular way to describe the behavior of the back-scattered radiation in the Thomson regime.

To this purpose Sec. II is devoted to the illustration of the kinematics in the center of mass reference frame and how a Lorentz transformation to the laboratory frame can bring to a simple exact analytical expression of the back-scattered photon energy at any scattering angle in the laboratory system, as a function of the electron energy $E_{e}$ and the incident photon energy $E_{L}$.

By recognizing the duality and interplay between the scattering angle and the single electron trajectory crossing angle in the interaction point due to the electron beam emittance, and applying a multivariate treatment to the photon energy distribution at small angles (i.e. $\gamma_{\mathrm{CM}} \theta<1$ ), we derive a complete expression of the bandwidth of the collimated photon beam within a collimation angle $\theta_{\max }$, set by the collimation system, as a function of the incoming beams features, i.e., the electron energy spread and transverse emittance, the laser photon frequency spread and phase front curvature, and the weak nonlinear effects represented by the laser parameter $a_{0}$. By using $\gamma_{\mathrm{CM}}$ instead of $\gamma$, the formula fully retrieves the effect of electron recoil to any extent, therefore is applicable to any ICSs regime. As anticipated, the bandwidth dependence on the electron beam emittance is generalized to $2\left(\gamma_{\mathrm{CM}} / \gamma\right)^{2}\left(\epsilon_{n} / \sigma_{x}\right)^{2}$, i.e. is basically not dependent on recoil in low recoil regimes (where $\gamma_{\mathrm{CM}}=\gamma$ ) while it scales like the inverse of recoil factor for large recoils (when $\gamma_{\mathrm{CM}} \ll \gamma$ ), showing a desensitivity of bandwidth from the electron beam emittance, a crucial feature that allows different strategies in large recoil ICSs for beam manipulation in order to maximize luminosity, as discussed further below.

Another surprising prediction is that bandwidth is largely independent on frequency spread of the incident photon beam in the deep recoil regime, that brings to a very interesting feature of deep recoil regime: the possibility to generate narrow bandwidth photon beams even using a broad bandwidth incident photon beam, and, at the same time, even over-focusing the electron beam (thanks to the suppressed dependence of bandwidth on electron beam emittance): as reported in Sec. III, numerical simulations nicely confirm this interesting prediction about a unique feature of deep recoil regime, that eventually leads to possible new options for ICSs designs.

These two new findings enhance the analytical description of ICSs with respect to previous works $[1,2,41,45,46]$ in predicting the behavior of the scattered photon beam phase space in deep recoil, as observed in numerical simulation of Ref. [25].

Furthermore, considering the expression of Klein-Nishina differential cross section we derive, through a luminosity treatment of the collision, an approximate expression for the number of photons emitted within a specific collimation angle $\theta_{\max }$, under the assumptions of $\gamma_{\mathrm{CM}} \theta_{\max }<1$, therefore also a simple expression for the spectral density (i.e. the number of photons normalized to absolute bandwidth) and brilliance of the emitted radiation beam.

Although our analysis is restricted to linear quantum model of the electron-photon collision, as previously said some of the nonlinear effects, e.g., those due to high intensity of the incident laser, are integrated in the model to some approximation. Extensive comparisons of the analytical formulas derived in Sec. II versus the results of 
Monte Carlo simulations are presented in Sec. III. We selected three different ICSs as paradigmatic of various regimes: (i) STAR I, a typical Thomson Source for x-ray generation in the $20-100 \mathrm{keV}$ range, devoted to radiological imaging of pre-clinical studies and cultural heritage studies: electron recoil effects are absolutely negligible in this case, where x-ray flux and moderate bandwidth are the key factors (hence maximum luminosity); (ii) ELI-NP-GBS II, a typical Inverse Compton source for nuclear photonics and photo-nuclear physics devoted to generate maximum spectral density photon beams in the 1-20 MeV energy range: here electron recoil is small but non negligible (actually larger than the requested narrow bandwidth); (iii) XFELO $-\gamma$ [25], a FEL based Inverse Compton source for hadronic physics experiments generating up to $7 \mathrm{GeV}$ photons by backscattering a $12 \mathrm{keV}$ FEL beam by a $7 \mathrm{GeV}$ electron beam generated by ERL: here electron recoil is dominant and strongly affects the bandwidth and intensity of the photon beam. The comparison between analytical predictions and simulation results underline impressively the predicted effect of decreasing the sensitivity of bandwidth to the electron beam emittance by a factor scaling with the inverse of recoil, in such a way that a stronger focusing of the electron beam can be applied without spoiling the bandwidth. As is well known this is not possible in low recoil regimes. The last section is devoted to comments and conclusions.

\section{THEORY}

Let us consider the collision between an electron and a counterpropagating photon of energy respectively $E_{e}$ and $E_{L}$ in the laboratory frame (LAB). We set $c=\hbar=1$. The energy $E_{L}^{\prime}$ of the colliding photon in the electron rest frame is given by (relativistic Doppler effect)

$$
E_{L}^{\prime}=E_{L} \gamma\left(1-\underline{\beta} \cdot \underline{e}_{k}\right)
$$

where $\beta$ is the velocity of the electron, $\underline{e}_{k}$ is the direction of propagation of the photon, $\gamma=E_{e} / M_{e}$ and $M_{e}=$ $0.511 \mathrm{MeV} / \mathrm{c}^{2}$. For an ultrarelativistic electron colliding head-on with a photon, the formula simplifies in $E_{L}^{\prime} \simeq$ $2 \gamma E_{L}$. The energy available in the center of mass (CM) of the electron-photon system is

$$
E_{\mathrm{CM}}=\sqrt{P^{2}}=\sqrt{2 E_{e} E_{L}-2\left(\underline{p}_{e} \cdot \underline{k}\right)+M_{e}^{2}}
$$

where $P=\left\{E_{e}+E_{L}, \underline{p}_{e}+\underline{k}\right\}$ and $\underline{p}_{e}, \underline{k}$ the electron and laser photon momenta respectively. Assuming $E_{e} \gg E_{L}$ and $\gamma \gg 1$,

$$
\gamma_{\mathrm{CM}}=\frac{E_{\mathrm{tot}}^{L A B}}{E_{\mathrm{CM}}} \simeq \frac{E_{e}+E_{L}}{\sqrt{4 E_{e} E_{L}+M_{e}^{2}}} .
$$

Once we define the parameter representing the recoil of the electron in the collision as

$$
X=\frac{4 E_{e} E_{L}}{M_{e}^{2}},
$$

we can write $E_{\mathrm{CM}} \simeq M_{e} \sqrt{1+X}$ and $\gamma_{\mathrm{CM}} \simeq \gamma / \sqrt{1+X}$.

We suppose the electron moves along the positive direction of the $z$ axis in LAB. In the center of mass frame $\mathrm{CM}$ the modulus of the momentum of electron and backscattered photon are

$$
p_{e}^{*}=E_{\mathrm{ph}}^{*}=\frac{E_{\mathrm{CM}}^{2}-M_{e}^{2}}{2 E_{\mathrm{CM}}}=\frac{X M_{e}}{2 \sqrt{1+X}},
$$

showing that there is no threshold for this reaction (as expected, it is a scattering) so that the electron recoil can be arbitrarily small $(*$ denotes the particles' momenta and energies in their $\mathrm{CM}$ reference frame). A Lorentz transformation to the LAB gives the energy of the scattered photon as a function of the $\mathrm{CM}$ scattering angle $\theta^{*}\left(\theta^{*}\right.$ calculated with respect to the $z$ axis):

$E_{\mathrm{ph}}=E_{\mathrm{ph}}^{*} \gamma_{\mathrm{CM}}\left(1+\beta_{\mathrm{CM}} \cos \theta^{*}\right)=4 E_{L} \gamma_{\mathrm{CM}}^{2} \frac{\left(1+\beta_{\mathrm{CM}} \cos \theta^{*}\right)}{2}$,

which exhibits

$$
\left\{\begin{array}{l}
E_{\mathrm{ph}}^{\max }=E_{\mathrm{ph}}\left(\theta^{*}=0\right)=4 E_{L} \gamma_{\mathrm{CM}}^{2}=\frac{4 \gamma^{2} E_{L}}{1+X} \\
E_{\mathrm{ph}}^{\min }=E_{\mathrm{ph}}\left(\theta^{*}=\pi\right)=E_{L}
\end{array}\right.
$$

and we can therefore write

$$
X=\frac{E_{\mathrm{ph}}^{\max }}{E_{e}-E_{\mathrm{ph}}^{\max }} .
$$

Actually, at very small angles in the $\mathrm{CM}$, the corresponding laboratory angle is $\theta=\theta^{*} \sqrt{1+X} / 2 \gamma$, and the photon momentum at small angles around the electron propagation axis (back-scattering close to the Compton edge) is given by

$$
E_{\mathrm{ph}}=4 \gamma_{\mathrm{CM}}^{2} E_{L}\left(1-\gamma_{\mathrm{CM}}^{2} \theta^{2}\right)
$$

as well known from the description of the collimated spectral characteristics of Compton sources, that are typically operated with relativistic electrons in very small recoil regime as specified by $X \ll 1$.

Since

$$
\begin{aligned}
\tan \theta & =\left[\frac{\sin \theta^{*}}{\gamma_{\mathrm{CM}}\left(\beta_{\mathrm{CM}}+\cos \theta^{*}\right)}\right], \\
\cos \theta^{*} & = \begin{cases}\frac{\sqrt{1+\tan ^{2} \theta}-\beta_{\mathrm{CM}} \gamma_{\mathrm{CM}}^{2} \tan ^{2} \theta}{1+\gamma_{\mathrm{CM}}^{2} \tan ^{2} \theta} & \text { if } \theta \leq \pi / 2, \\
\frac{-\sqrt{1+\tan ^{2} \theta}-\beta_{\mathrm{CM}} \gamma_{\mathrm{CM}} \tan ^{2} \theta}{1+\gamma_{\mathrm{CM}}^{2} \tan ^{2} \theta} & \text { if } \theta>\pi / 2 .\end{cases}
\end{aligned}
$$

Equations (6) and (11) fully specify in a simple analytical form the energy of the scattered photons as a function of $E_{e}, E_{L}$ and $\theta$. 
In the following we analyze the dependence of the emitted photons' relative bandwidth $\Delta E_{\mathrm{ph}} / E_{\mathrm{ph}}$ from the laser and the electron beam parameters, which are: $\gamma$ the Lorentz factor, $\Delta \gamma / \gamma$ the relative energy spread, $\epsilon_{n}$ the normalized emittance and $\sigma_{x}$ the rms spot size at interaction point of the electron beam, $\Delta E_{L} / E_{L}$ the laser bandwidth, $\lambda_{0}$ the laser wavelength, $w_{0}$ the laser focal spot size, $M^{2}$ the beam quality factor and the laser parameter $a_{0}$. We improve and generalize the formula described in Refs. [3,36,41,45] by taking into account the effect given by the electron recoil on the emitted radiation: the use of $\gamma_{\mathrm{CM}}$ instead then $\gamma$ extends the validity of the equation to any recoil regime.

As in the above mentioned references, we consider a Gaussian phase space distribution for the electron beam and for the laser pulse while the resulting shape of the photon spectrum is determined by the energy-angle correlation described by Eqs. (6) and (11).

We define the acceptance angle as

$$
\Psi=\gamma_{\mathrm{CM}} \theta_{\max }
$$

and the term

$$
\bar{P}=\gamma_{\mathrm{CM}} \frac{\sqrt{2} \epsilon_{x}}{\sigma_{x}}=\frac{\sqrt{2} \epsilon_{n}}{\sigma_{x} \sqrt{1+X}}
$$

where $\sqrt{2} \epsilon_{n} / \sigma_{x}$ represents the normalized rms transverse momentum of the electron beam which coincides with $\bar{P}$ at low recoil. Instead $\bar{P}$ is reduced by a factor $\gamma_{\mathrm{CM}} / \gamma \simeq \sqrt{X}$ when the recoil is large. The relative bandwidth of the emitted radiation is given by

$$
\frac{\Delta E_{\mathrm{ph}}}{E_{\mathrm{ph}}} \simeq \sqrt{\left[\frac{\Psi^{2} / \sqrt{12}}{1+\Psi^{2}}+\frac{\bar{P}^{2}}{1+\sqrt{12} \bar{P}^{2}}\right]^{2}+\left[\left(\frac{2+X}{1+X}\right) \frac{\Delta \gamma}{\gamma}\right]^{2}+\left(\frac{1}{1+X} \frac{\Delta E_{L}}{E_{L}}\right)^{2}+\left(\frac{M^{2} \lambda_{0}}{2 \pi w_{0}}\right)^{4}+\left(\frac{a_{0}^{2} / 3}{1+a_{0}^{2} / 2}\right)^{2}}
$$

where in case of a laser Gaussian both in longitudinal and transverse directions

$$
a_{0}=6.8 \frac{\lambda_{0}}{w_{0}} \sqrt{\frac{U_{L}(J)}{\sigma_{t}(p s)}}
$$

with $U$ the energy of the laser and $\sigma_{t}$ the rms laser pulse length.

We note that Eq. (14) is based on a fourth order expansion in the acceptance angle $\Psi$ : this approach limits the validity of the formula to angles $\Psi<1$.

The number of scattered photons per second is given by

$$
\mathcal{N}=\mathcal{L} \sigma=\frac{N_{e} N_{L} r}{2 \pi\left(\sigma_{x}^{2}+\sigma_{L}^{2}\right)} \sigma
$$

where $\mathcal{L}$ is the luminosity,

$$
\begin{aligned}
\sigma= & \frac{2 \pi r_{e}^{2}}{X}\left[\frac{1}{2}+\frac{8}{X}-\frac{1}{2(1+X)^{2}}\right. \\
& \left.+\left(1-\frac{4}{X}-\frac{8}{X^{2}}\right) \log (1+X)\right]
\end{aligned}
$$

is the total unpolarized Compton cross section [49], $N_{e}, N_{L}$ are the number of incoming electrons and photons, $r$ is the repetition rate of the collisions, and $\sigma_{x}, \sigma_{L}=w_{0} / 2$ are the rms spot size radius at the interaction point of the electron and photon beams respectively. The value of $\sigma$ varies between the classical limit $X \rightarrow 0$ and the ultra-relativistic limit $X \rightarrow \infty$ as presented in Eq. (18) where $\sigma_{T}=0.67$ barn represents the total Thomson cross section [50].

$$
\left\{\begin{array}{l}
\lim _{X \rightarrow 0} \sigma=\frac{8 \pi r_{e}^{2}}{3}(1-X)=\sigma_{T}(1-X) \\
\lim _{X \rightarrow \infty} \sigma=\frac{2 \pi r_{e}^{2}}{X}\left(\log X+\frac{1}{2}\right)
\end{array}\right.
$$

In practical units,

$$
\mathcal{N}=4.2 \times 10^{8} \frac{\sigma U_{L}(\mathrm{~J}) Q(\mathrm{pC}) r}{\sigma_{T} E_{L}(\mathrm{eV})\left[\sigma_{x}^{2}(\mu \mathrm{m})+\sigma_{L}^{2}(\mu \mathrm{m})\right]} .
$$

By using the Compton differential cross section [49] in the approximation $\Psi<1$, we obtain the analytical expression to estimate $\mathcal{N}^{\Psi}$, the number of photons in acceptance angle $\Psi$, and the spectral density $S$ :

$$
\begin{gathered}
\mathcal{N}^{\Psi}=6.25 \times 10^{8} \frac{U_{L}(\mathrm{~J}) Q(\mathrm{pC}) r}{E_{L}(\mathrm{eV})\left[\sigma_{x}^{2}(\mu \mathrm{m})+\sigma_{L}^{2}(\mu \mathrm{m})\right]} \\
\times \frac{\left(1+\sqrt[3]{X} \Psi^{2} / 3\right) \Psi^{2}}{\left[1+(1+X / 2) \Psi^{2}\right]\left(1+\Psi^{2}\right)}, \\
S=\frac{\mathcal{N}^{\Psi}}{\sqrt{2 \pi} 4 E_{L} \gamma_{\mathrm{CM}}^{2} \frac{\Delta E_{\mathrm{ph}}}{E_{\mathrm{ph}}}} .
\end{gathered}
$$

The rms source spot size is

$$
\sigma_{s}=\frac{\sigma_{x} \sigma_{L}}{\sqrt{\sigma_{x}^{2}+\sigma_{L}^{2}}}
$$

and the emittance of the emitted radiation is

$$
\epsilon_{\gamma}=\sigma_{s} \frac{\theta_{\max }}{\sqrt[4]{12} \sqrt[9]{1+X}}
$$

The peak brilliance is defined as

$$
B^{\text {peak }}=\frac{\mathcal{N}^{\Psi}}{(2 \pi)^{3} \epsilon_{\gamma}^{2} \sigma_{t}^{\gamma} \frac{\Delta E_{\mathrm{ph}}}{E_{\mathrm{ph}}}[0.1 \%] r}
$$

with $\sigma_{t}^{\gamma}$ the rms duration value of the emitted $\gamma$ photons. 
The average brilliance on one second is instead given by

$$
B^{\text {ave }}=\frac{\mathcal{N}^{\Psi}}{(2 \pi)^{\frac{5}{2}} \epsilon_{\gamma}^{2} \frac{\Delta E_{\mathrm{ph}}}{E_{\mathrm{ph}}}[0.1 \%]} .
$$

\section{SIMULATIONS}

We will benchmark some of the formulas in the previous section against the simulated values obtained in STAR, ELI-NP-GBS, and XFELO- $\gamma$ cases. The simulations have been performed by means of the Monte Carlo codes CAIN and CMCC $[33,35]$.

From the simulations data, $\Delta E_{\mathrm{ph}} / E_{\mathrm{ph}}$ is calculated as the rms value of the distributions divided by their mean value:

$$
\begin{aligned}
\frac{\Delta E_{\mathrm{ph}}}{E_{\mathrm{ph}}} & =\frac{\sqrt{\left\langle E_{\mathrm{ph}}^{2}\right\rangle-\left\langle E_{\mathrm{ph}}\right\rangle^{2}}}{\left\langle E_{\mathrm{ph}}\right\rangle} \\
\left\langle E_{\mathrm{ph}}\right\rangle & =\frac{1}{N} \sum_{i=1, N} E_{\mathrm{ph}_{i}} \\
\left\langle E_{\mathrm{ph}}^{2}\right\rangle & =\frac{1}{N} \sum_{i=1, N} E_{\mathrm{ph}_{i}}^{2}
\end{aligned}
$$

\begin{tabular}{|c|c|c|c|c|c|c|c|c|}
\hline Case & $\begin{array}{c}\mathrm{Q} \\
\mathrm{e}(\mathrm{nC})\end{array}$ & $\begin{array}{c}E_{e} \\
(\mathrm{MeV})\end{array}$ & $\begin{array}{c}\Delta \gamma / \gamma \\
\left(10^{-3}\right)\end{array}$ & $\begin{array}{c}\epsilon_{n} \\
(\mu \mathrm{m} \operatorname{rad})\end{array}$ & $\begin{array}{c}\sigma_{p_{x}} \\
(\mathrm{keV})\end{array}$ & $\begin{array}{c}\sigma_{x} \\
(\mu \mathrm{m})\end{array}$ & $\begin{array}{c}\lambda_{0} \\
(\mu \mathrm{m})\end{array}$ & $\begin{array}{l}\mathrm{U}_{L} \\
(\mathrm{~J})\end{array}$ \\
\hline $\begin{array}{l}\text { A } \\
\text { B }\end{array}$ & 1 & 65 & 5 & (2) & $\begin{array}{l}34 \\
68\end{array}$ & $\begin{array}{c}15 \\
7.5\end{array}$ & 1 & 0.2 \\
\hline \multicolumn{9}{|c|}{$X=0.00123$} \\
\hline
\end{tabular}

TABLE I. Interaction parameters for STAR. $\sigma_{L}=15 \mu \mathrm{m}, \sigma_{t}=$ $1 \mathrm{ps}$ and $\sigma_{p_{x}}$ electron beam rms transverse momentum $(\mathrm{keV})$.

TABLE II. Interaction parameters for ELI-NP-GBS. $\sigma_{L}=$ $14 \mu \mathrm{m}, \sigma_{t}=1.5 \mathrm{ps}$.

\begin{tabular}{lcccccccc}
\hline \hline & $\begin{array}{c}\mathrm{Q} \\
\text { Case }(\mathrm{pC})\end{array}$ & $\begin{array}{c}E_{e} \\
(\mathrm{MeV})\end{array}$ & $\begin{array}{c}\Delta \gamma / \gamma \\
\left(10^{-4}\right)\end{array}$ & $\begin{array}{c}\epsilon_{n} \\
(\mu \mathrm{m} \mathrm{rad})\end{array}$ & $\begin{array}{c}\sigma_{p_{x}} \\
(\mathrm{keV})\end{array}$ & $\begin{array}{c}\sigma_{x} \\
(\mu \mathrm{m})\end{array}$ & $\begin{array}{c}\lambda_{0} \\
(\mathrm{~nm})\end{array}$ & $\begin{array}{c}\mathrm{U}_{L} \\
(\mathrm{~J})\end{array}$ \\
\hline $\mathrm{C}$ & 250 & 311.65 & 7 & \begin{tabular}{c}
0.5 \\
\multicolumn{7}{c}{$X=0.011$}
\end{tabular} \\
\hline \hline
\end{tabular}

TABLE III. Interaction parameters XFELO- $\gamma$. Gaussian distribution for position and momentum of the electrons. $N_{L}=2 \times 10^{10}, \sigma_{p_{x}}=5.4 \mathrm{keV}, \sigma_{L}=8.9 \mu \mathrm{m}$ and $\sigma_{t}=0.85 \mathrm{ps}$.

\begin{tabular}{lccccccc}
\hline \hline & $\begin{array}{c}\mathrm{Q} \\
\text { Case }(\mathrm{pC})\end{array}$ & $\begin{array}{c}E_{e} \\
(\mathrm{GeV})\end{array}$ & $\begin{array}{c}\Delta \gamma / \gamma \\
\left(10^{-4}\right)\end{array}$ & $\begin{array}{c}\epsilon_{n} \\
(\mu \mathrm{m} \mathrm{rad})\end{array}$ & $\begin{array}{c}\sigma_{x} \\
(\mu \mathrm{m})\end{array}$ & $\begin{array}{c}\lambda_{0} \\
(\mathrm{~nm})\end{array}$ & $\begin{array}{c}\mathrm{U}_{L} \\
(\mu \mathrm{J})\end{array}$ \\
\hline $\mathrm{D}$ & & & & & & 1000 & 0.00397 \\
$\mathrm{E}$ & 40 & 7 & 2 & 0.082 & 7.7 & 10 & 0.397 \\
$\mathrm{~F}$ & & & & & & 0.1 & 39.7 \\
\hline \hline
\end{tabular}

TABLE IV. Interaction parameters XFELO $-\gamma$.

\begin{tabular}{lccccccc}
\hline \hline & & $E_{\mathrm{CM}}$ & & & $1 / \gamma$ & $1 / \gamma_{\mathrm{CM}}$ & \\
Case & $X$ & $(\mathrm{MeV})$ & $\gamma$ & $\gamma_{\mathrm{CM}}$ & $(\mu \mathrm{rad})$ & $(\mu \mathrm{rad})$ & $\mathcal{N}$ \\
\hline $\mathrm{D}$ & 0.1328 & 0.542 & & 12915 & & 77 & 0.34 \\
$\mathrm{E}$ & 13.28 & 1.93 & 13700 & 3626.94 & 73 & 275 & $6.3 \times 10^{-2}$ \\
$\mathrm{~F}$ & 1328 & 18.36 & & 375.73 & & 2660 & $1.7 \times 10^{-3}$ \\
\hline \hline
\end{tabular}

where $E_{\mathrm{ph}_{i}}$ is the energy of the $i$ th emitted photon and $N$ the number of photons in the considered set.

In the following examples we always consider $\mathcal{N}$ and $\mathcal{N}^{\Psi}$ [Eqs. (19), (20)] per shot, i.e. $r=1$ and the electron
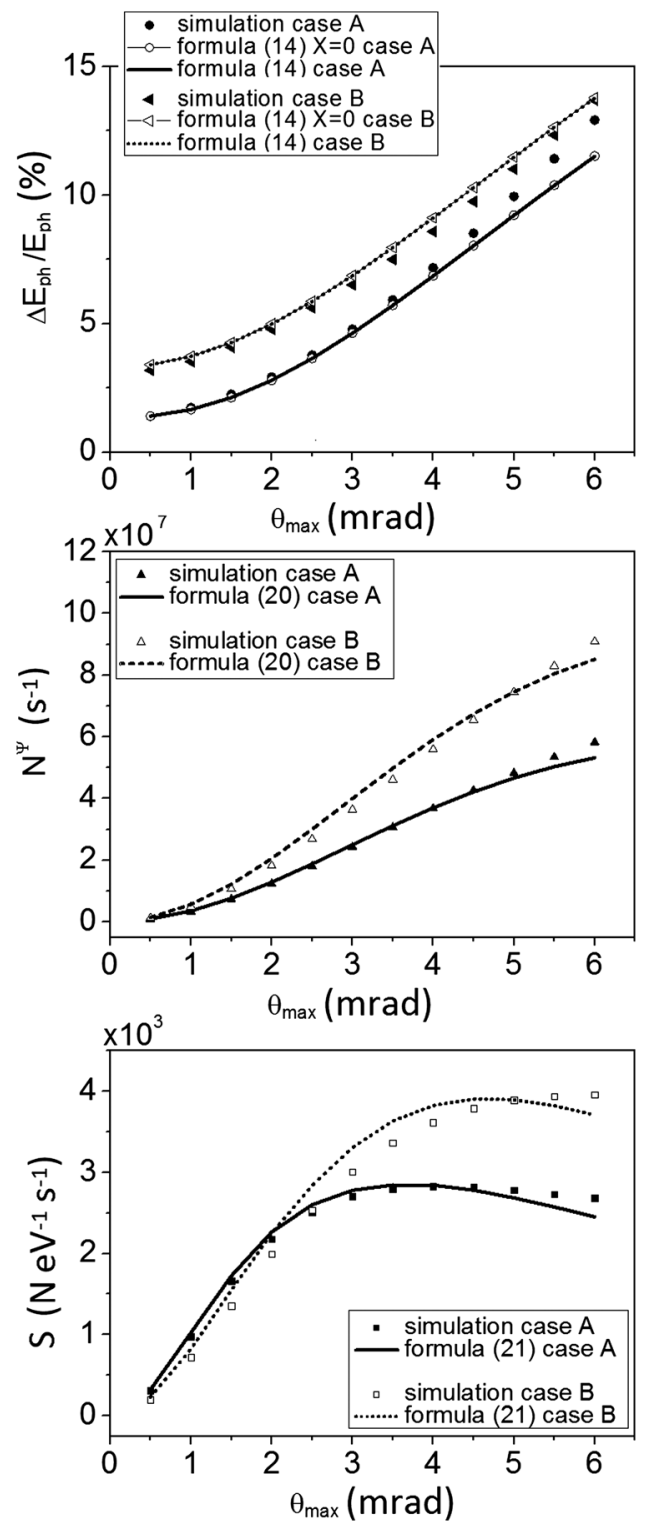

FIG. 1. Cases A and B: $\Delta E_{\mathrm{ph}} / E_{\mathrm{ph}}(\%)$ from CAIN simulation vs formula (14) without and with $X$ correction, $N^{\Psi}\left(\mathrm{s}^{-1}\right)$ number of photons from CAIN simulation vs formula (20), $S\left(\mathrm{~N} \mathrm{eV}^{-1}\right.$ $\left.\mathrm{s}^{-1}\right)$ spectral density per shot $(r=1)$ from CAIN simulation vs formula (21) as a function of $\theta_{\max }(\mathrm{mrad})$. 


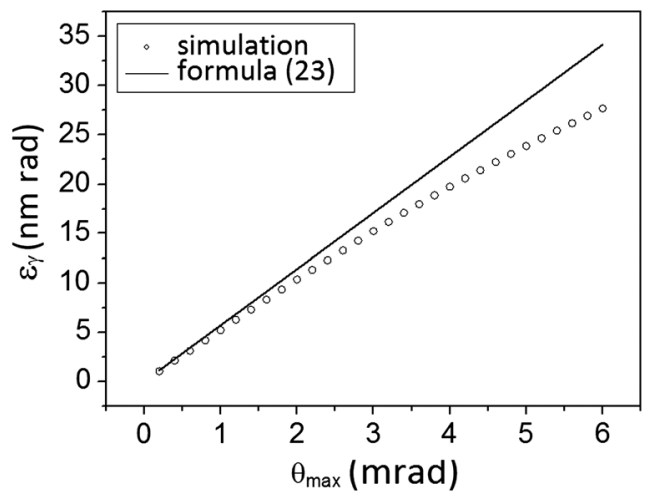

FIG. 2. Case A: $\epsilon_{\gamma}$ (nm rad) value from CAIN simulation vs formula (23) as a function of $\theta_{\max }(\mathrm{mrad})$.
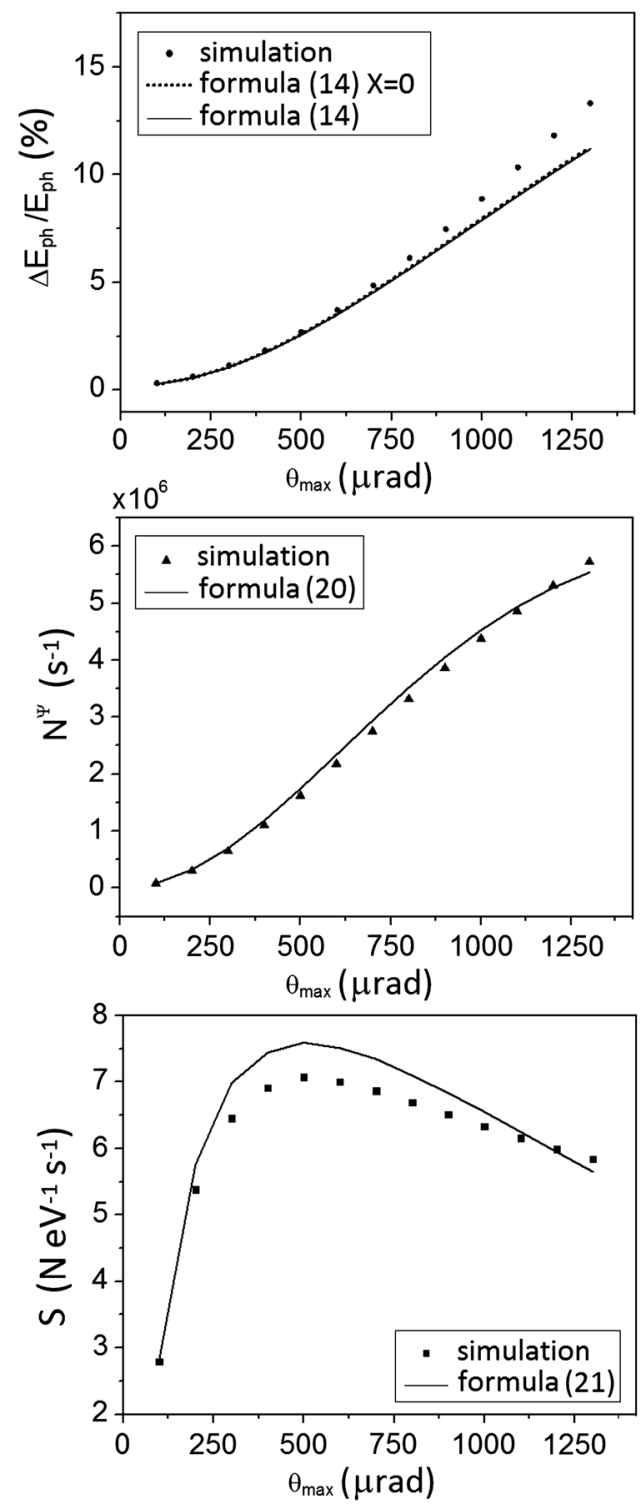

FIG. 3. Case C: $\Delta E_{\mathrm{ph}} / E_{\mathrm{ph}}(\%)$ value from CAIN simulation vs formula (14) without and with $X$ correction. $N^{\Psi}\left(\mathrm{s}^{-1}\right)$ number of photons in $\theta_{\max }$ : simulated values vs formula (20). $S\left(\mathrm{~N} \mathrm{eV}^{-1} \mathrm{~s}^{-1}\right)$ spectral density per $\operatorname{shot}(r=1)$ : simulated values vs formula (21).

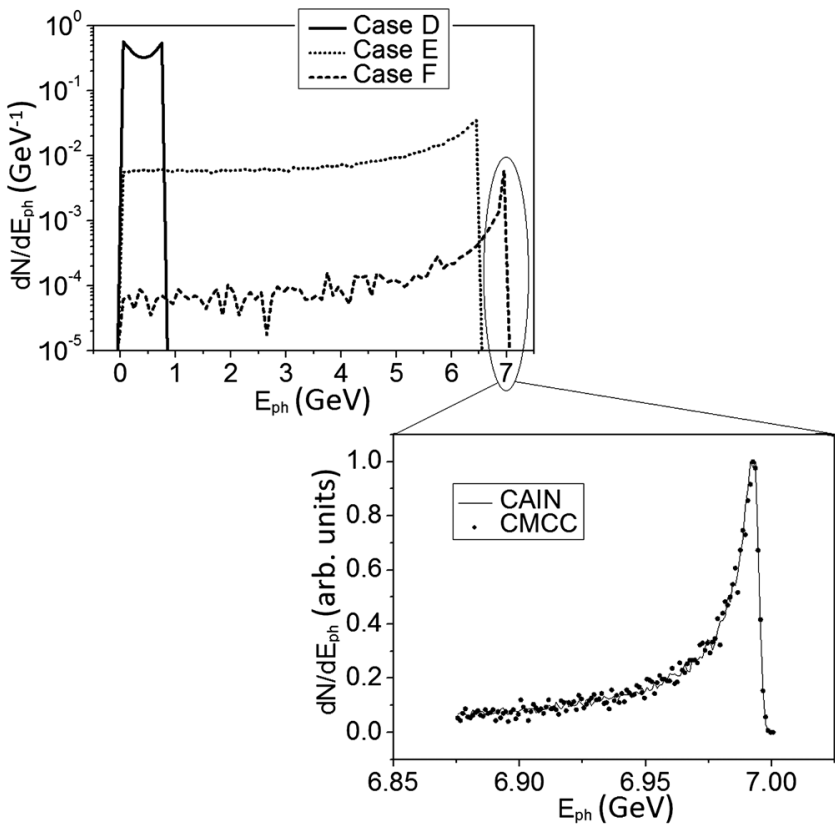

FIG. 4. Spectrum of the emitted photons for the three XFELO- $\gamma$ cases, CMCC simulations. Close-up for case $\mathrm{F}$ with comparison between CAIN and CMCC results.

beam rms length equal to the laser pulse one $\sigma_{z}=c \sigma_{t}$. Furthermore the collisions are perfectly head-on (collision angle $\alpha=0$ ) and the beam diffraction throughout the interaction region is negligible so that Eq. (16) is applicable because the luminosity is not spoiled by hourglass effects.

\section{A. STAR}

The Southern European Thomson source for Applied Research (STAR), under construction at the University of Calabria, is a typical example of Thomson source. We report in Table I the interaction parameters: the very low $X$ value enables a classical approach to this source study. Two different focusing of the electron beam have been considered: in both cases the impact of the recoil parameter on the bandwidth value is negligible (see Fig. 1). In case B, $\Delta E_{\mathrm{ph}} / E_{\mathrm{ph}}$ value is higher than in case $\mathrm{A}$ at small collimation angles ( $\bar{P}$ is doubled) and also the number of photons $\mathcal{N}^{\Psi}$ and the spectral density $S$ are higher in case B at any $\theta_{\max }$.

Figure 2 exhibits a very good agreement between formula (23) and the value of the emitted photon beam emittance given by the CAIN simulations.

\section{B. ELI-NP-GBS}

The Extreme Light Infrastructure Nuclear Physics Gamma Beam System (ELI-NP-GBS) is a linear machine based on the collision of an intense high power $\mathrm{Yb}$ :Yag J-class laser and a high brightness electron beam with a tunable energy up to $750 \mathrm{MeV}$. The main specifications 

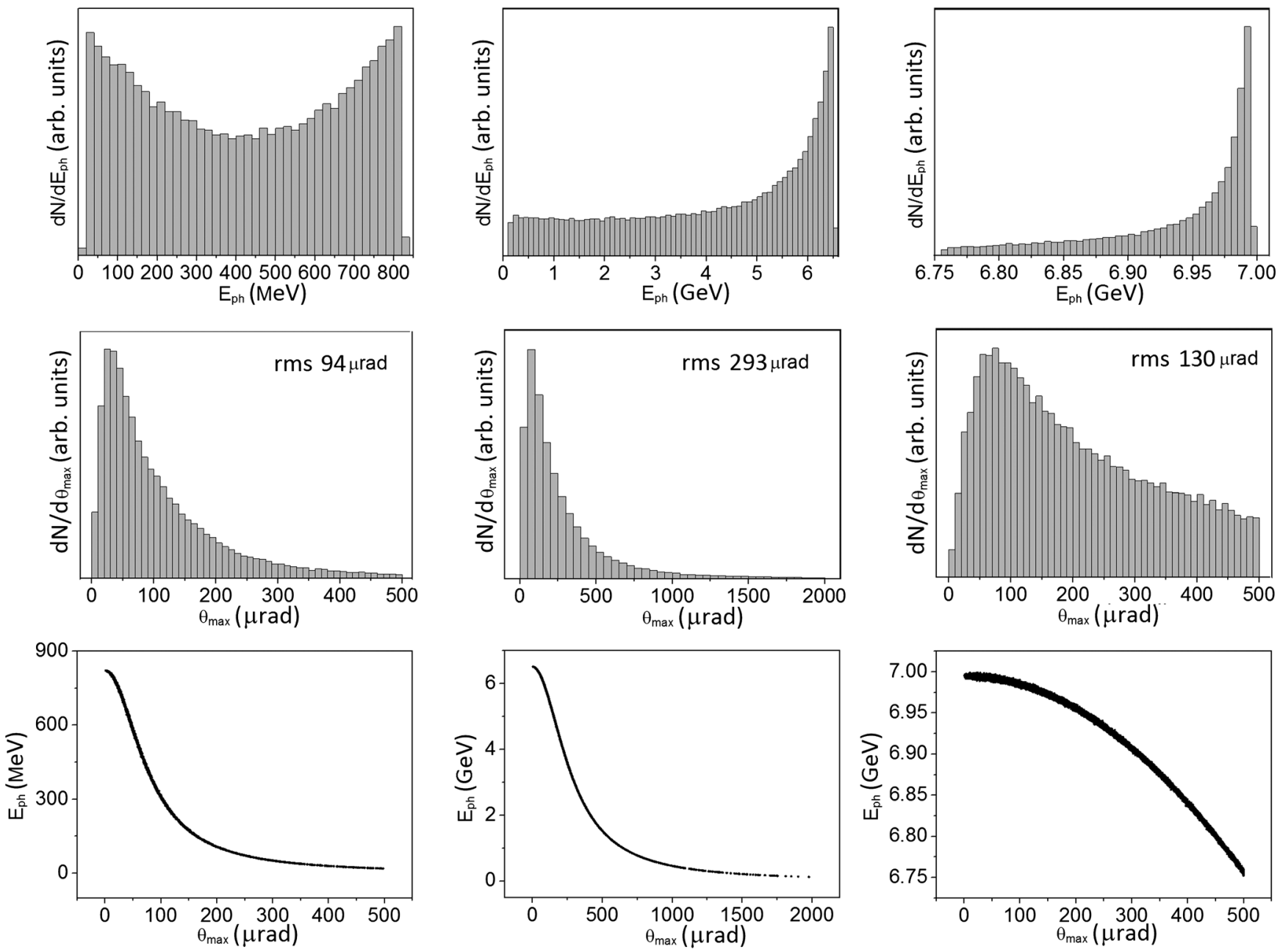

FIG. 5. Emitted photons features, CAIN simulations. First line spectrum, second line angular distribution, third line energy as a function of $\theta_{\max }(\mu \mathrm{rad})$. Case D left column, case E middle column, case F right column.

of the Compton Source are: photon energy tunable in the 0.2-19.5 MeV energy range, rms relative bandwidth smaller than $0.5 \%$ and, since $r=3200$, spectral density lager than $5 \times 10^{3}$ photons $/(\mathrm{s} \cdot \mathrm{eV})$, with source spot sizes smaller than $100 \mu \mathrm{m}$ and linear polarization of the gammaray beam larger than $95 \%$. Moreover the peak brilliance of the emitted $\gamma$ beam is expected to be larger than $10^{19}$ photons $/\left(\mathrm{s} \cdot \mathrm{mm}^{2} \cdot \mathrm{mrad}^{2} \cdot 0.1 \%\right)$.

The interaction parameters we used in the following simulations are reported in Table II. The recoil parameter is quite low, nevertheless its impact is not negligible since the bandwidth value request is highly demanding. Figure 3 suggests a $\theta_{\max } \ll 1 / \gamma_{\mathrm{CM}}=1220 \mu \mathrm{rad}$ to obtain $\Delta E_{\mathrm{ph}} / E_{\mathrm{ph}}=0.005$ and it shows the spectral density peaked around an angle of about $400 \mu \mathrm{rad}$. Due to the error propagation (the spectral density scales like the ratio between the number of photons in a certain angle and the associated bandwidth value), the bottom graph of Fig. 3 shows a small discrepancy of the order of few $\%$ between the simulated and the predicted values around the peak.

\section{XFELO- $\gamma$}

The XFELO- $\gamma$ design described in [25] considers the collision between a $7 \mathrm{GeV}$ electron beam and three possible photon beam energies covering a wide range of recoil regimes: a $1.239 \mathrm{eV}$ photon beam leads to a low recoil parameter $X=0.1328$, an intermediate case at $123.9 \mathrm{eV}$ sets $X=13.28$ and a very high recoil regime is reached at $12.39 \mathrm{keV}$ (the interaction parameters are summarized in Tables III and IV). The emitted radiation spectra as given by CAIN and CMCC are reported for all the cases in Fig. 4 and they are analyzed in detail in Fig. 5.

The low $X$ case (D) exhibits the typical Thomson spectrum shape, $\gamma \simeq \gamma_{\mathrm{CM}}$ and half of the emitted photons are contained in the small $1 / \gamma_{\mathrm{CM}} \simeq 1 / \gamma$ angle. Figure 6 shows a good agreement between the low recoil case and the classical treatment $(X=0)$, Fig. 7 reports a very good agreement between simulations and formulas concerning the bandwidth calculation at small angles for different $\sigma_{x}$ and normalized emittance values. 

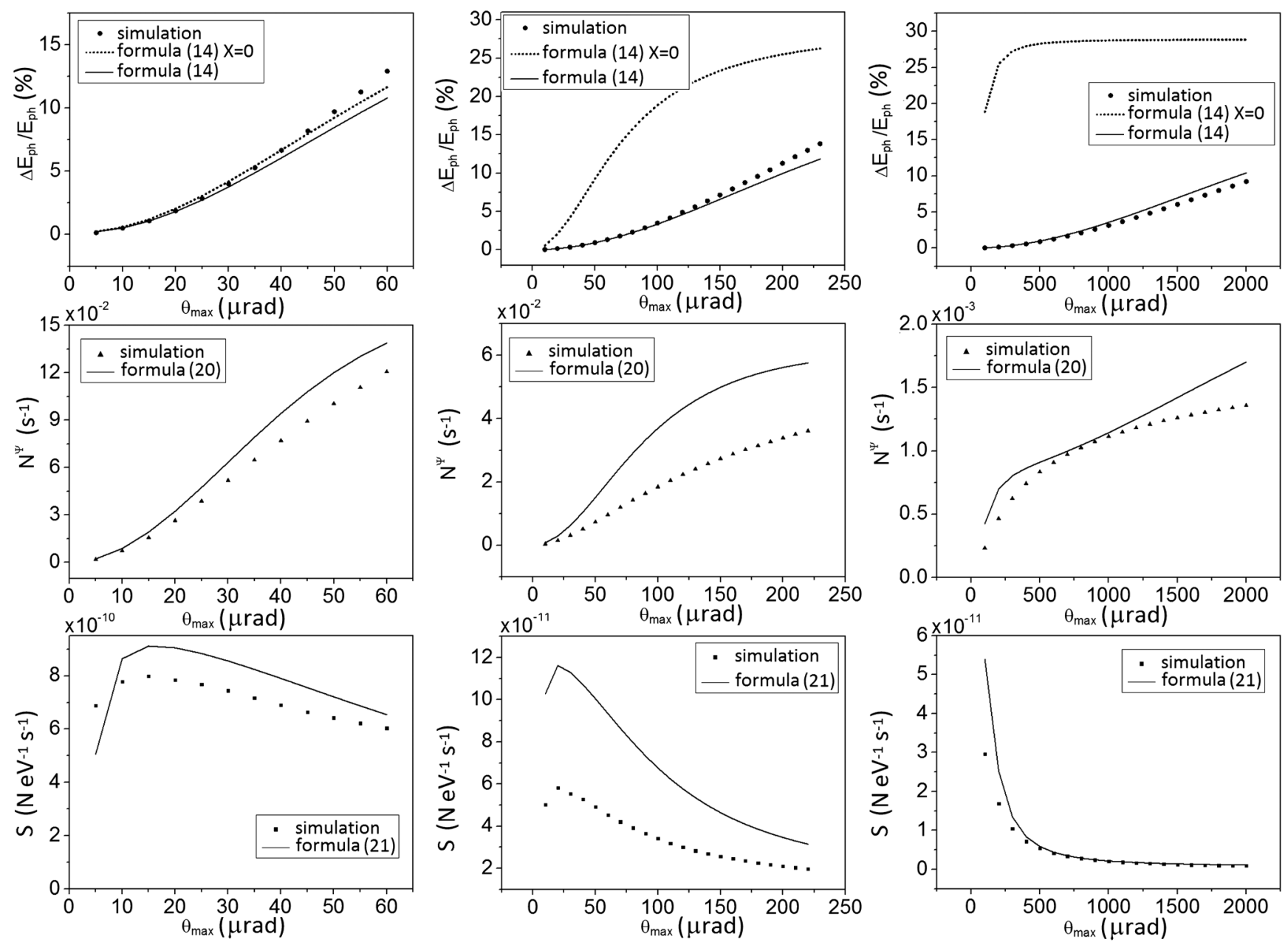

FIG. 6. $\Delta E_{\mathrm{ph}} / E_{\mathrm{ph}}(\%)$ value from CAIN simulation vs formula (14) without and with $X$ correction. $N^{\Psi}\left(\mathrm{s}^{-1}\right)$ number of photons in $\theta_{\max }$ : simulated values vs formula (20). $S\left(\mathrm{~N} \mathrm{eV}^{-1} \mathrm{~s}^{-1}\right)$ spectral density per shot $(r=1)$ : simulated values vs formula (21). Case D left column, case E middle column and case F right column.

The spectrum in the medium recoil case E (Fig. 5) shows an appreciable asymmetry toward the high energies given by the strong asymmetry of the differential cross section. In this intermediate case the $X$ contribution to the bandwidth value calculation is not negligible as shown in Fig. 6 (top line, central graph).

The most unusual regime is the one at very large recoil: here the $X$ contribution is fundamental in the $\Delta E_{\mathrm{ph}} / E_{\mathrm{ph}}$ calculation (right column, uppermost graph of Fig. 6). The energy spectrum is peaked around the incoming electron beam energy (Figs. 4, 5). Most of the incoming electron energy is transferred to the photons which are emitted on a wider rms $\theta$ with respect to the low recoil case. This large recoil regime shows some very peculiar characteristics not present in the other cases: the bandwidth value becomes insensitive to some of the incoming beams features such as the electron beam emittance and the laser bandwidth. As presented in Fig. 8, the variation of $\sigma_{x}$ does not affect the bandwidth value and even a big increase in the $\epsilon_{n}$ value corresponds to a really modest increase of the emitted radiation bandwidth. In the same way a huge $10 \%$ increase of $\Delta E_{L} / E_{L}$ leads to a small $7 \times 10^{-5} \Delta E_{\mathrm{ph}} / E_{\mathrm{ph}}$ broadening (Fig. 9 left graph).

The emittance of the scattered photon beam described by Eq. (23) is in agreement with the simulations (see Fig. 9 right graph), therefore also the values of peak and average brilliance predicted by formulas (24), (25) are consistent with the Monte Carlo results.

As presented by Fig. 6, Eq. (14) gives an extremely good agreement in all the considered recoil regimes and it sets a great accuracy improvement with respect to the same formula at $X=0$. On the other hand, formula (20) [and consequently formula (21)] loses its accuracy in the intermediate recoil case: due to the complexity of the performed integral an asymptotic approach has been used, therefore the validity of Eq. (20) is guaranteed for $X<1$ and $X \gg 1$. In the intermediate recoil region $(1<X<100)$ the formula can be used to estimate the order of magnitude but a complete simulation is needed in order to have an accurate prediction on the number of photons in the acceptance angle. 

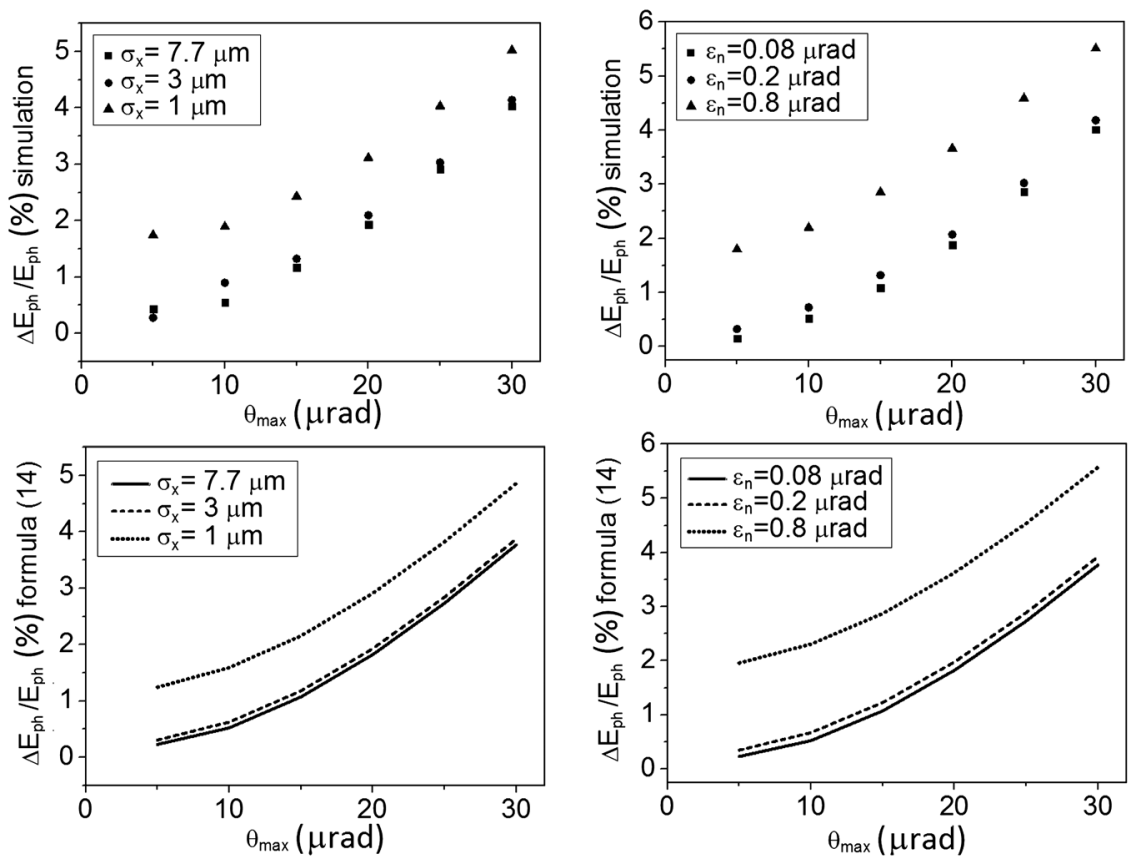

FIG. 7. Case D: $\Delta E_{\mathrm{ph}} / E_{\mathrm{ph}}(\%)$ as a function of $\theta_{\max }(\mu \mathrm{rad})$ for $\sigma_{x}=7.7,3,1 \mu \mathrm{m}$ at $\epsilon_{n}=0.082 \mu \mathrm{m}$ rad (left column) and for $\epsilon_{n}=0.08,0.2,0.8 \mu \mathrm{m} \mathrm{rad}$ at $\sigma_{x}=7.7 \mu \mathrm{m}$ (right column). Simulations (top line) and formula (14) (bottom line).
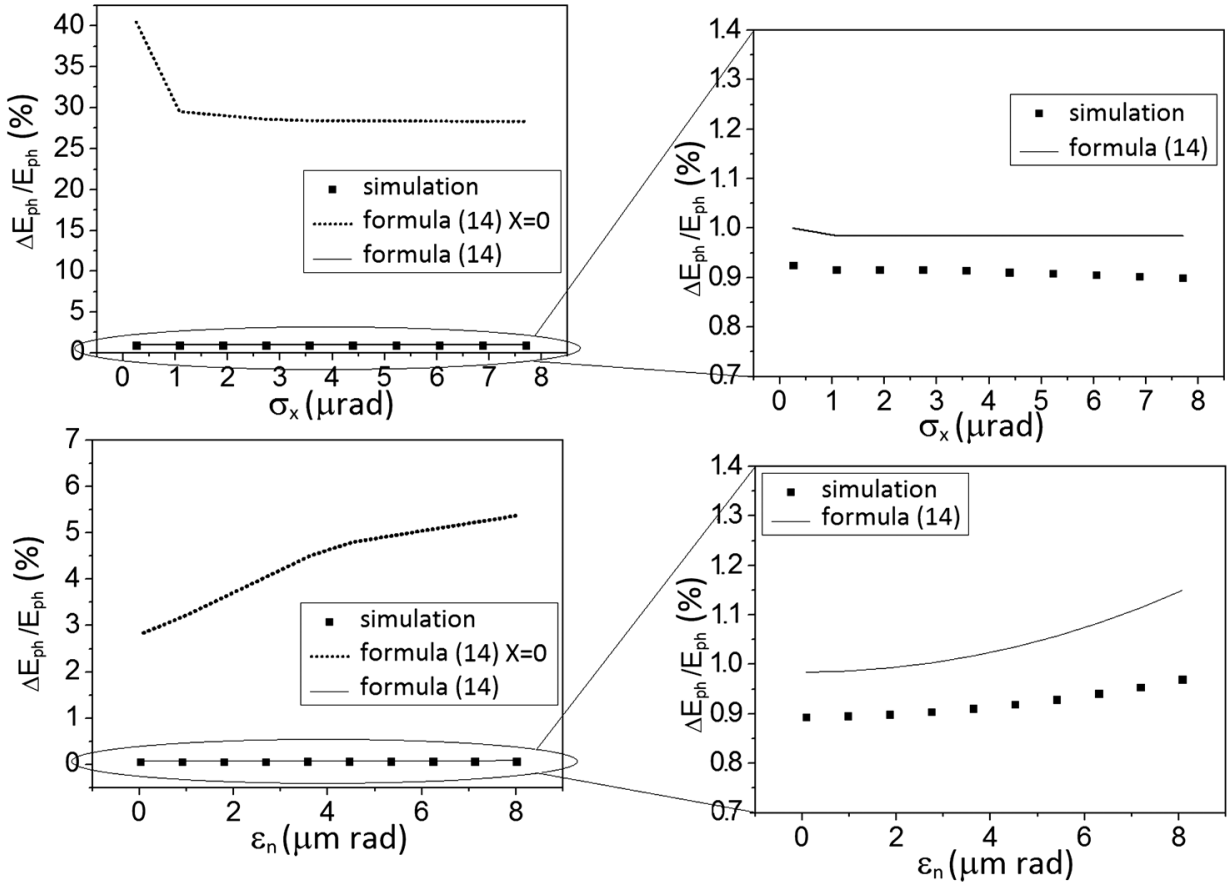

FIG. 8. Case F: $\Delta E_{\mathrm{ph}} / E_{\mathrm{ph}}(\%)$ value from CAIN simulation vs formula (14) without and with $X$ correction as a function of $\sigma_{x}(\mu \mathrm{m})$ (top line) and of $\epsilon_{n}\left(\mu \mathrm{m}\right.$ rad) (bottom line) at fixed $\theta_{\max }=500 \mu \mathrm{rad}$. 

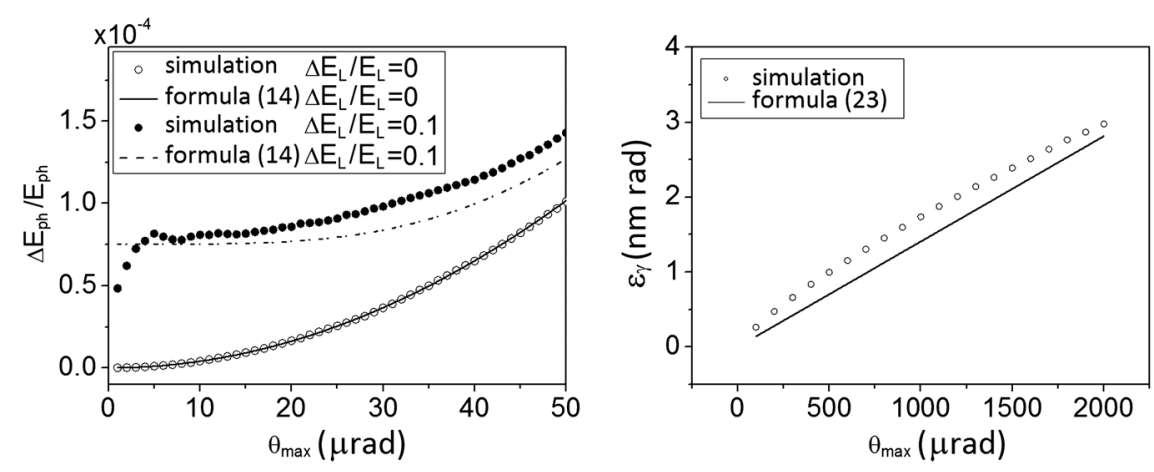

FIG. 9. Case F. Left graph: $\Delta E_{\mathrm{ph}} / E_{\mathrm{ph}}$ value from CMCC simulation vs formula (14) as a function of $\theta_{\max }$ ( $\mu \mathrm{rad}$ ), for $\Delta E_{L} / E_{L}=0$ and $\Delta E_{L} / E_{L}=0.1$. Right graph: $\epsilon_{\gamma}(\mathrm{nm} \mathrm{rad})$ value from CAIN simulation vs formula (23) as a function of $\theta_{\max }(\mu \mathrm{rad})$.

\section{CONCLUSIONS}

We derived simple analytical formulas able to predict with quite good accuracy the characteristics of $6 \mathrm{D}$ phase space distributions of the $\mathrm{X} / \gamma$ photon beams generated by back-scattering in inverse Compton sources. The formulas are strictly valid in the linear regime. Since most of the ICSs in operation, under construction and design, to be implemented as user facilities, are meant to be operated in linear or weak nonlinear regime, the set of formulas here presented constitutes a useful scheme of guidelines to help ICSs designers with quick predictions of their anticipated performances in terms of flux, bandwidth, spectral density, emittance, and brilliance of the photon beam.

In particular the formulas, derived by developing the kinematics in the center of mass reference system of the electron-photon collision, are valid for any value of the electron recoil, covering the whole range of energy of colliding electrons ( $\mathrm{MeV}$ to multi-GeV) and incident photons (from optical laser pulses to multi-keV FEL's), with the only restriction of relativistic electrons and incident photons with energy much smaller than the electron one. Under this respect, such a generalization to any value of the electron recoil represents an upgrade with respect to previous works.

Two somewhat new findings [25] that occur at large recoil, $X \gg 1$, have been theoretically explained throughout this paper: (i) a suppression of the bandwidth dependence on the electron beam emittance; (ii) a much weaker dependence of the bandwidth on the frequency spread of the incident photon beam. These effects are basically negligible in the usual regime of ICSs, not only the socalled Thomson regime for X-rays used for radiological imaging, but even those used for nuclear physics/photonics with $\mathrm{MeV}$-class photon beams. Only when higher energy incident photon beams are considered, like for instance with X-ray FEL's, the effects come to play with full deployment, and can be exploited in a strategic way to optimize the design of recoil dominated ICSs. Allowing for instance an overfocusing of the electron beam to maximize luminosity without spoiling the photon beam bandwidth, which is not allowed in low recoil ICSs due to the dependence of bandwidth on the electron beam emittance (i.e., the rms electron beam transverse momentum). Also the use of a broad-band incident photon beam would be possible in large recoil ICSs, without spoiling the photon bandwidth, thanks to the large suppression applied by the large recoil factor.

The analysis reported here is valid for unpolarized beams and a perfectly head-on collision. The generalization to small nonzero collision angle is done by correcting the recoil factor to

$$
X=\frac{2 E_{e} E_{L}}{M_{e}^{2}}(1+\cos \alpha)
$$

where $\alpha$ is the (small) collision angle. The correction to luminosity due to nonzero collision angle, in the approximation $\alpha \ll 1$, can be taken care by multiplying the luminosity by the correction factor $\delta_{\alpha}[51]$

$$
\delta_{\alpha}=\frac{1}{\sqrt{1+\frac{\alpha^{2}\left(\sigma_{z}^{2}+c^{2} \sigma_{t}^{2}\right)}{4\left(\sigma_{x}^{2}+\sigma_{L}^{2}\right)}}} .
$$

In particular note that formula ([51]) describing the number of emitted photons, as well as formulas (19), (20), (21), (24), (25) derived from it, are strictly valid in case of negligible diffraction effects in the collision.

[1] G. A. Krafft and G. Priebe, Compton sources of electromagnetic radiation, Rev. Accel. Sci. Techol. 03, 147 (2010).

[2] C. Sun and Y. K. Wu, Theoretical and simulation studies of characteristics of a Compton light source, Phys. Rev. ST Accel. Beams 14, 044701 (2011).

[3] O. Adriani et al., ELI-NP-GBS Technical Design Report, http://arxiv.org/ftp/arxiv/papers/1407/1407.3669.pdf.

[4] D. Micieli, I. Drebot, A. Bacci, E. Milotti, V. Petrillo, M. Rossetti Conti, A. R. Rossi, E. Tassi, and L. Serafini, 
Compton sources for the observation of elastic photon-photon scattering events, Phys. Rev. Accel. Beams 19, 093401 (2016).

[5] C. Curatolo, F. Broggi, and L. Serafini, Phase space analysis of secondary beams generated in hadron-photon collisions, Nucl. Instrum. Methods Phys. Res., Sect. A 865, 128 (2017).

[6] I. V. Pogorelsky et al., Demonstration of $8 \times 10^{18}$ photons/ second peaked at $1.8 \AA$ in a relativistic Thomson scattering experiment, Phys. Rev. ST Accel. Beams 3, 090702 (2000).

[7] M. Bech, O. Bunk, C. David, R. Ruth, J. Rifkin, R. Loewen, R. Feidenhans'1, and F. Pfeiffer, Hard X-ray phase-contrast imaging with the Compact Light Source based on inverse Compton X-rays, J. Synchrotron Radiat. 16, 43 (2009).

[8] R. Kuroda, H. Toyokawa, M. Yasumoto, H. IkeuraSekiguchi, M. Koike, K. Yamada, T. Yanagida, T. Nakajyo, F Sakai, and K. Mori, Quasi-monochromatic hard X-ray source via laser Compton scattering and its application, Nucl. Instrum. Methods Phys. Res., Sect. A 637, S183 (2011).

[9] K. Achterhold, M. Bech, S. Schleede, G. Potdevin, R. Ruth, R. Loewen, and F. Pfeiffer, Monochromatic computed tomography with a compact laser-driven X-ray source, Sci. Rep. 3, 1313 (2013).

[10] Y. Du et al., Generation of first hard X-ray pulse at Tsinghua Thomson Scattering X-ray Source, Rev. Sci. Instrum. 84, 053301 (2013).

[11] A. Jochmann et al., High Resolution Energy-Angle Correlation Measurement of Hard X Rays from Laser-Thomson Backscattering, Phys. Rev. Lett. 111, 114803 (2013).

[12] S. Chen et al., MeV-Energy X Rays from Inverse Compton Scattering with Laser-Wakefield Accelerated Electrons, Phys. Rev. Lett. 110, 155003 (2013).

[13] W. Luo, H. B. Zhuo, Y. Y. Ma, Y. M. Song, Z. C. Zhu, T. P. $\mathrm{Yu}$, and M. Y. Yu, Attosecond Thomson-scattering X-ray source driven by laser-based electron acceleration, Appl. Phys. Lett. 103, 174103 (2013).

[14] E. Eggl et al., X-ray phase-contrast tomosynthesis of a human ex vivo breast slice with an inverse Compton $\mathrm{x}$-ray source, Eur. J. Phys. 116, 68003 (2016).

[15] C. Vaccarezza et al., The SPARC_LAB Thomson source, Nucl. Instrum. Methods Phys. Res., Sect. A 829, 237 (2016).

[16] M. Niiyama, Recent results from LEPS and prospects of LEPS II at SPring-8, Nucl. Phys. A914, 543 (2013).

[17] X. Wang, L. Wen, and H. Bosong, Adjustable LCS $\gamma$ Source SINAP-III, Nucl. Phys. Rev. 29, 253 (2012).

[18] R. Hajima et al., Generation of laser Compton scattered gamma-rays from a 150-MeV microtron, in Proc. 4th Int. Particle Accelerator Conf. (IPAC'13), Shanghai, China (2013), p. 3645.

[19] Z. Huang and R. D. Ruth, Laser-Electron Storage Ring, Phys. Rev. Lett. 80, 976 (1998).

[20] R. Loewen, Stanford University SLAC-Report No. 632, 2003.

[21] R. Loewen and R. D. Ruth, The Compact Light Source: A Miniature Synchrotron producing $X$-rays via Inverse Compton Scattering, in High-Brightness Sources and Light-Driven Interactions, OSA technical Digest (online) (Optical Society of America, 2016), paper ES5A.2.
[22] A. Ovodenko et al., High duty cycle inverse Compton scattering X-ray source, Appl. Phys. Lett. 109, 253504 (2016).

[23] A. Bacci et al., Status of the STAR project, in Proceedings of 7th International Particle Accelerator Conference (IPAC'16), Busan, Korea (2016), paper TUPOW004, p. 1747.

[24] A. Bacci et al., Electron Linac design to drive bright Compton back-scattering gamma-ray sources, J. Appl. Phys. 113, 194508 (2013).

[25] R. Hajima and M. Fujiwara, Narrow-band GeV photons generated from an x-ray free-electron laser oscillator, Phys. Rev. Accel. Beams 19, 020702 (2016).

[26] T. Akagi et al., Narrow-band photon beam via laser Compton scattering in an energy recovery linac, Phys. Rev. Accel. Beams 19, 114701 (2016).

[27] S. H. Park, Spatial distribution and polarization of $\gamma$-rays generated via Compton backscattering in the Duke/OK-4 storage ring FEL, Nucl. Instrum. Methods Phys. Res., Sect. A 475, 425 (2001).

[28] A. P. Tonchev, M. Boswell, C. R. Howell, H. J. Karwowski, J. H. Kelley, W. Tornow, and Y. K. Wu, The high intensity $\gamma$-ray source $(\mathrm{HI} \gamma \mathrm{S})$ and recent results, Nucl. Instrum. Methods Phys. Res., Sect. B 241, 170 (2005).

[29] A. Variola, J. Haissinski, A. Loulergue, and F. Zomer, ThomX Technical Design Report, 2014.

[30] D. Alesini, I. Chaikovska, S. Guiducci, C. Milardi, A. Variola, M. Zobov, and F. Zomer, DAФNE $\gamma$-rays factory, IEEE Trans. Nucl. Sci. 63, 913 (2016).

[31] B. E. Carlsten, F. L. Krawczyk, J. W. Lewellen, Q. R. Marksteiner, D. C. Nguyen, and N. A. Yampolsky, High repetition-rate inverse Compton scattering $\mathrm{x}$-ray source driven by a free electron laser, J. Phys. B 47, 234012 (2014).

[32] Y. K. Wu, Progress using an FEL oscillator for Compton Scattering, http://accelconf.web.cern.ch/AccelConf/ FEL2014/talks/wea01_talk.pdf (2014).

[33] K. Yokoya, User manual of CAIN, version 2.40, http://lcdev .kek.jp/yokoya/CAIN/Cain242/CainMan242.pdf (2009).

[34] W. Luo, H. B. Zhuo, Y. Y. Ma, X. H. Yang, N. Zhao, and M. Y. Yu, Ultrashort-pulse $\mathrm{MeV}$ positron beam generation from intense Compton-scattering $\gamma$-ray source driven by laser wakefield acceleration, Laser Part. Beams 31, 89 (2013).

[35] C. Curatolo, Ph.D. thesis, Università degli Studi di Milano, 2016, https://air.unimi.it/handle/2434/358227.

[36] P. Tomassini et al., Linear and nonlinear thomson scattering for advanced X-ray sources in PLASMONX, IEEE Trans. Plasma Sci. 36, 1782 (2008).

[37] N. D. Powers, I. Ghebregziabher, G. Golovin, C. Liu, S. Chen, S. Banerjee, J. Zhang, and D. P. Umstadter, Quasi-monoenergetic and tunable X-rays from a laserdriven Compton light source, Nat. Photonics 8, 28 (2014).

[38] A. D. Debus, M. Bussmann, M. Siebold, A. Jochmann, U. Schramm, T. E. Cowan, and R. Sauerbrey, Travelingwave Thomson scattering and optical undulators for highyield EUV and X-ray sources, Appl. Phys. B 100, 61 (2010). 
[39] V. Petrillo, G. Dattoli, I. Drebot, and F. Nguyen, Compton scattered X-gamma rays with orbital momentum, Phys. Rev. Lett. 117, 123903 (2016).

[40] F. Albert et al., Characterization and applications of a tunable, laser-based, $\mathrm{MeV}$-class Compton-scattering $\gamma$-ray source, Phys. Rev. ST Accel. Beams 13, 070704 (2010).

[41] V. Petrillo et al., Photon flux and spectrum of $\gamma$-rays Compton sources, Nucl. Instrum. Methods Phys. Res., Sect. A 693, 109 (2012).

[42] I. Chaikovska and A. Variola, Equilibrium energy spread and emittance in a Compton ring: An alternative approach, Phys. Rev. ST Accel. Beams 17, 044004 (2014).

[43] A. P. Potylitsyn and A. M. Kolchuzhkin, Spectral characteristics of Compton backscattering sources. Linear and nonlinear modes, Nucl. Instrum. Methods Phys. Res., Sect. B 355, 246 (2015).

[44] V. Petrillo et al., Polarization of x-gamma radiation produced by a Thomson and Compton inverse scattering, Phys. Rev. ST Accel. Beams 18, 110701 (2015).

[45] L. Serafini et al., High intensity $\mathrm{X} / \gamma$ photon beams for nuclear physics and photonics, EPJ Web Conf. 117, 05002 (2016).
[46] G. A. Krafft, E. Johnson, K. Deitrick, B. Terzić, R. Kelmar, T. Hodges, W. Melnitchouk, and J. R. Delayen, Laser pulsing in linear Compton scattering, Phys. Rev. Accel. Beams 19, 121302 (2016).

[47] W. J. Brown and F. V. Hartemann, Three-dimensional time and frequency-domain theory of femtosecond x-ray pulse generation through Thomson scattering, Phys. Rev. ST Accel. Beams 7, 060703 (2004).

[48] P. Cardarelli, M. Gambaccini, M. Marziani, E. Bagli, V. Petrillo, A. Bacci, C. Curatolo, I. Drebot, and C. Vaccarezza, Monte Carlo simulation of a collimation system for low-energy beamline of ELI-NP Gamma Beam System, Nucl. Instrum. Methods Phys. Res., Sect. B 355, 237 (2015).

[49] V. Berestetskii, E. Lifshitz, and L. Pitaevskii, Quantum Electrodynamics. Landau and Lifshitz, Course of Theoretical Physics (Pergamon Press, New York, 1982), Vol. 4.

[50] J. D. Jackson, Classical Electrodynamics (John Wiley \& Sons, Inc., New York, 1998).

[51] B. Muratori, CERN, SL-AP LHC Project Note Report No. 301rev, 2002. 